\title{
Lactate: a multifunctional signaling molecule
}

\section{Tae-Yoon Lee}

Department of Microbiology, Yeungnam University College of Medicine, Daegu, Korea

Received: December 22, 2020

Revised: January 15, 2021

Accepted: January 25, 2021

\section{Corresponding author:}

Tae-Yoon Lee, MD, PhD

Department of Microbiology,

Yeungnam University College of

Medicine, 170 Hyeonchung-ro,

Nam-gu, Daegu 42415, Korea

Tel: +82-53-640-6941

Fax: +82-53-653-6628

E-mail:doxr7p@gmail.com
Since its discovery in 1780, lactate has long been misunderstood as a waste by-product of anaerobic glycolysis with multiple deleterious effects. Owing to the lactate shuttle concept introduced in the early 1980s, a paradigm shift began to occur. Increasing evidence indicates that lactate is a coordinator of whole-body metabolism. Lactate is not only a readily accessible fuel that is shuttled throughout the body but also a metabolic buffer that bridges glycolysis and oxidative phosphorylation between cells and intracellular compartments. Lactate also acts as a multifunctional signaling molecule through receptors expressed in various cells and tissues, resulting in diverse biological consequences including decreased lipolysis, immune regulation, anti-inflammation, wound healing, and enhanced exercise performance in association with the gut microbiome. Furthermore, lactate contributes to epigenetic gene regulation by lactylating lysine residues of histones, accounting for its key role in immune modulation and maintenance of homeostasis.

Keywords: Glycolysis; Homeostasis; Lactate; Lactate receptor; Lactate shuttle; Lactylation; Oxidative; Warburg effect

\section{Introduction}

Single-celled organisms, such as bacteria and fungi, are put under pressure to reproduce as soon as they encounter nutrient-rich environments. Their metabolism control systems have evolved to sense available nutrients and transfer the necessary carbon, nitrogen, and energy to generate building blocks for cell growth. When starved, the cells cease to produce biomass and begin adapting their metabolism to draw maximum energy from available resources to cope with nutrient depletion [1].

In multicellular organisms, uncontrolled proliferation is prevented as mammalian cells cease to take up nutrients from the environment unless otherwise stimulated by growth factors. Genetically mutated cancer cells, on the other hand, are non-dependent on such external stimuli and may show increased uptake and metabolism of nutrients, promoting cell survival and proliferation [1]. Oncogenic mutations often result in a high-rate uptake of nutrients, particularly glucose, which is required for cell growth and prolifera- tion [2]. In 1923, Otto Warburg found that when glucose was added to cancer tissue slices in Ringer's solution, they continuously produced lactate in the presence of oxygen [3,4]. His attempt was to understand why cancer cells preferred glucose fermentation over oxidative phosphorylation (OXPHOS), even in the presence of ample oxygen. This aerobic glycolysis occurs not only in cancer cells but also in exercising muscle cells, activated immune cells, and virally infected cells. A glycolytic increase in lactate concentration is found in local tissues under various disease conditions, such as sepsis, infections, inflammatory diseases, autoimmune diseases, and cancer.

Lactate has long been regarded as a by-product of anaerobic respiration. Increasing evidence, however, indicates that lactate is essential for energy and redox homeostasis. More recent data suggest that lactate is a multifunctional signaling molecule with its own receptor and plays an important role in communication between cells and tissues. The aim of this review was to provide knowledge on lactate, including its general physiology and historical paradigm 
changes, and to summarize the recent advances that highlight the regulatory role of lactate in diverse biological processes in both physiological and pathological conditions.

\section{Physiology of lactate}

Almost 99\% of lactic acid is dissociated into lactate anions $\left(\mathrm{La}^{-}\right)$ and protons $\left(\mathrm{H}^{+}\right)$within the physiological $\mathrm{pH}$ range. Therefore, in this review, $\mathrm{La}^{-}$will be referred to as lactate. Lactate is mainly composed of $\mathrm{L}(+)$ enantiomer ( $\mathrm{L}$-form) as detected in the sera of healthy humans [5]. Serum D-lactate concentration ranges from 0.013 to $0.2 \mathrm{mM}$, as opposed to that of L-lactate which may range from slightly above $1.14 \mathrm{mM}$ at rest, $15 \mathrm{mM}$ after an intense exercise, and $30 \mathrm{mM}$ in some cancer tissues [6]. The daily production of lactate in resting humans is estimated to be approximately 20 $\mathrm{mmol} / \mathrm{kg} /$ day [7]. In adults with an average body weight of $70 \mathrm{~kg}$, approximately $1,400 \mathrm{mmol}$ of lactate is produced daily from muscle (25\%), skin (25\%), brain (20\%), red blood cells (20\%), and intestines $(10 \%)$ [8].

Lactate is produced by the reduction of pyruvate by lactate dehydrogenase (LDH) during glycolysis under anaerobic or aerobic conditions.

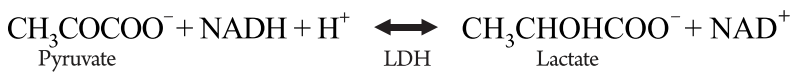

$\mathrm{LDH}$ is a tetrameric enzyme that catalyzes the interconversion of pyruvate and lactate with the complementary interconversion of a reduced form of nicotinamide adenine dinucleotide (NADH) to $\mathrm{NAD}^{+}$. The two most common subunits of $\mathrm{LDH}$ are the LDHM (or M subunit) and LDHH (or $\mathrm{H}$ subunit) proteins that are encoded by $L D H A$ and $L D H B$ genes, respectively. These subunits combine to form five isoenzymes: LDH-1 (4H), LDH-2 (3H1M), LDH-3 (2H2M), LDH-4 (1H3M), and LDH-5 (4M) [9]. LDH-1 is usually called LDHB since it is composed of four "H" LDHB subunits. LDHB preferentially converts lactate to pyruvate and is expressed mainly in the heart $(\mathrm{H})$. In contrast, $\mathrm{LDH}$ 5 is usually called LDHA since it is composed of four "M" LDHA subunits. LDHA preferentially converts pyruvate to lactate and is expressed mainly in the skeletal muscle (M) [10]. Heterogenous LDHs (LHH-2 to LDH-4) exhibit subunit-dependent intermediate enzyme activity. The remaining two subunits, $\mathrm{LDH}-\mathrm{C}$ and $\mathrm{LDH}-\mathrm{Bx}$, are specific to the testes and peroxisomes, respectively. In lactate-producing glycolysis, LDHA is the most important enzyme.

\section{Lactate paradigm changes}

Lactate has long been considered a waste end product of anaerobic glycolysis [11-14]. The development of acidosis has traditionally been explained by lactate overproduction during high-intensity exercises or severe disease states such as sepsis and septic shock [15]. A high level of serum lactate has been regarded as a predictive factor of muscle fatigue, often associated with tissue hypoxia and poor clinical outcomes [16].

The early 1980s was marked by a drastic change in the lactate paradigm. The idea that lactate is not responsible for acidosis started gaining acceptance, as reviewed by Robergs et al. [17]. According to the theory, intermediate acids of glycolysis have a low pKa; hence they all exist in their base form. The first intermediate acid of glycolysis, 3-phosphoglyceric acid, is present as 3-phosphoglycerate. This signifies that the main form of glycolytic metabolites is not acid (pyruvic acid or lactic acid) but a base (pyruvate or lactate). For example, at physiological $\mathrm{pH}(\sim 6.4$ to 7.1$)$, lactic acid, due to its very low acid dissociation constant $(\mathrm{pKa}, \sim 3.86)$, dissociates immediately into lactate and hydrogen ion $\left(\mathrm{H}^{+}\right)$. This indicates that lactic acid does not exist in living organisms. In fact, lactate does not decrease metabolic acidosis, but rather removes $\mathrm{H}^{+}$ from the cytosol and creates $\mathrm{NAD}^{+}$molecules, electron acceptors that are used for adenosine triphosphate (ATP) generation to maintain glycolysis $[18,19]$.

How, then, is lactate removed? To maintain the serum concentration range of 1 to $2 \mathrm{mM}, 60$ to $120 \mathrm{mmol}$ of lactate needs to be removed from the blood every hour [20]. Lactate is usually removed immediately from various tissues, for example, skeletal muscles, or it may be released and taken up by exercising muscle, heart, brain, kidney, and liver. Lactate may also be metabolized either by direct oxidation or transformation into glucose. Lactate transformation into glucose by the liver or kidney is known as the Cori cycle or gluconeogenesis [21]. Lactate can also be removed via its oxidation into pyruvate, which involves an entry into the tricarboxylic acid (TCA) cycle needed for ATP production [22,23].

\section{Lactate shuttles}

As the product of glycolysis and the substrate for OXPHOS or gluconeogenesis, lactate can be regarded as the link between different metabolic pathways. In 1985, George Brooks [24] proposed the lactate shuttle hypothesis. Lactate shuttles and metabolic interplay are now recognized to mediate redox and energy homeostasis not only between cells, tissues, and at the whole organism level but also at an intracellular level between cell compartments, such as cytosol-mitochondria or cytosol-peroxisome shuttle. The early lactate 
shuttle reported in various cells, tissues, and organs, including muscle, heart, and liver [24-27], has now extended to those of sperm [28], adipose tissue [29], the brain [30], and the lungs [31-33]. Approximately half of the available lactate is removed through oxidation at rest, and up to $80 \%$ during exercise [34].

In contracting skeletal muscle, for example, lactate production and utilization occur simultaneously. It has been proposed that myocytes have two metabolic compartments, one for glycolysis (cytoplasm) and the other for oxidative metabolism (mitochondria) [35]. According to this concept, cytosolic lactate enters mitochondria via lactate transporters (intracellular lactate shuttle) and is oxidized to pyruvate through the mitochondrial lactate oxidation complex at the mitochondrial inner membrane, composed of mitochondrial LDH, CD147, and cytochrome oxidase [35]. In the myocardium, lactate uptake increases during conditions such as exercise, $\beta$-adrenergic stimulation, fast-pacing, and shock. Lactate may account for up to $60 \%$ of the cardiac oxidation substrate [36]. In the human brain, lactate accounts for approximately $7 \%$ of the energy requirement during rest, which increases up to $25 \%$ during exercise [37]. The lactate from the blood is either oxidized by neurons or converted and stored as glycogen. The lactate from glycolysis of the stored glycogen in astrocytes is transported into neurons (referred to as astrocyte-neuronal lactate shuttle) and oxidized to pyruvate and utilized by the TCA cycle $[38,39]$.

\section{Lactate transporters}

Lactate is transported across cytoplasmic and intracellular compartments by several monocarboxylate transporters (MCTs) that perform proton-lactate symport. Among the $14 \mathrm{MCTs}$ that have been identified to date, MCT1-4 are better characterized and have been shown to mediate the proton-linked transport of monocarboxylates such as lactate, pyruvate, acetoacetate, and $\beta$-hydroxybutyrate. MCT1 (also known as SLC16A1) is widely distributed and is usually involved in the import of lactate, whereas MCT4 (also known as SLC16A3) is expressed in highly glycolytic cells or tissues, such as white skeletal muscle, astrocytes, cancer cells, and white blood cells, and is mainly involved in lactate export $[39,40]$. In addition to MCTs, sodium-coupled lactate transport is carried out by the high-affinity transporter SLC5A8 or the low-affinity transporter SLC5A12 as was initially reported in the kidney [41]. The expression of SLC5A12 has also been reported in $\mathrm{CD}^{+} \mathrm{T}$ cells [42].

\section{Lactate receptor}

Lactate is a ligand for an orphan G-protein coupled receptor 81
(GPR81), now termed hydroxycarboxylic acid receptor 1 (HCAR-1 or HCA1) [39]. HCA1 is predominantly expressed in the adipose tissue. Other tissues and organs also express HCA1, although to a lesser degree, in the skeletal muscle, liver, spleen, kidney, and brain. In addition to the plasma membrane, $\mathrm{HCAl}$ is also detected in intracellular organelles, such as mitochondria. As expected from the broad presence of the lactate receptor $\mathrm{HCAl}$ in various tissues, increasing evidence has shown that lactate plays diverse roles in various pathophysiological conditions, including inflammation and cancer. HCA1-mediated lactate signaling may affect lipid metabolism [43], neuronal excitability changes [44], cellular development and survival $[45,46]$, and modulation of inflammatory responses $[47,48]$. Lactate generated by glycolytic cancer cells can also act on HCA1 expressed on non-cancer cells, including immune cells, endothelial cells, adipocytes, and fibroblasts in the tumor stroma. The final consequence of lactate-mediated activation of HCA1 on cells in the tumor mass is the facilitation of survival, growth, and metastasis of cancer cells via mechanisms that mediate increased angiogenesis, immune evasion, and chemoresistance [49].

Lactate signaling via HCA1 has been shown to work synergistically with insulin to decrease cellular concentration of cyclic adenosine monophosphate (cAMP) and lipolysis in the fed state, suggesting that HCA1 might be linked to obesity [29]. The mechanism of cAMP modulation includes attenuation of protein kinase A (PKA) signaling [50,51]. Lactate produced and released by inflammatory bone marrow neutrophils may induce mobilization via endothelial HCA1 signaling by inhibiting the expression of VE-cadherin in intercellular junctions of the vascular endothelium [52].

Lactate binding to HCA1 can also signal through a noncanonical, cAMP/PKA-independent pathway with arrestin beta 2 (ARRB2) as an adaptor protein, leading to the inhibition of tolllike receptor-4 (TLR-4)- and nucleotide-binding oligomerization domain (NOD)-like receptor pyrin domain-containing protein 3 (NLRP3) inflammasome-mediated induction of proinflammatory mediators such as interleukin (IL)-1 $\beta$ and IL-18 [47,48,53].

\section{Warburg effect}

Normal cells obtain ATP, an essential energy source for cell survival, from both glycolysis and mitochondrial OXPHOS. In 1923, Otto Warburg and Seigo Minami [3] found high rates of glycolysis in cultured tumor tissues, which were characterized by increased glucose uptake and excessive lactate production even in the presence of oxygen (aerobic glycolysis). They incubated slices of rat hepatoma in Ringer's solution and found an increase in lactate concentration calculated from the increase in $\mathrm{CO}_{2}$ during 30 minutes 
of incubation. The rate of lactate production from the hepatoma slice was 70 times greater than that in the normal liver, kidney, and heart tissue [54]. Warburg [4] also showed, in a rat model, that the artery feeding the tumor had lower levels of lactate and higher levels of glucose than the draining vein from the tumor tissue. This glucose-avid, lactate-producing behavior of tumor cells regardless of oxygen availability was named the "Warburg effect" by Efraim Racker in 1972 [55].

\section{Why cancer cells prefer the Warburg phenotype?}

Mitochondrial function is not impaired in Warburg cells, as described in many types of cancers; the TCA cycle is activated in breast cancer, glioblastoma, and non-small cell lung cancer cells [56]. Therefore, the Warburg effect is a metabolic shift from (not a defect of) OXPHOS to glycolysis. Glycolysis generates only two molecules of ATP per glucose molecule, which is much less efficient than OXPHOS (36 ATPs per glucose). Nevertheless, most cancer cells obtain approximately $60 \%$ of the total required ATP from glycolysis. The activation of glycolysis has several advantages for rapidly proliferating cancer cells. In addition to the faster ATP synthesis than OXPHOS, the activation of the glycolytic pathway allows cells to feed several pathways that contribute to macromolecular synthesis. These include the pentose phosphate pathway, which produces ribose for nucleotides and NADPH for reductive biosynthesis, the hexosamine pathway, which is required for protein glycosylation, serine-glycine-one-carbon metabolism, which feeds glutathione, nucleotides, and methylation reactions; and glycerol synthesis for the production of complex lipids [57].

\section{The mechanism of the metabolic shift from OXPHOS to glycolysis}

One of the key components of glycolytic activation and OXPHOS suppression is hypoxia-inducible factor $1 \alpha$ (HIF-1 $\alpha$ ) [58], which was originally identified as a critical transcription factor for cellular adaptation to hypoxic conditions. HIF-1 $1 \propto$ induces most of the glycolytic enzymes, including hexokinase 2, pyruvate kinase M2 (PKM2), and LDHA. HIF-1 $\alpha$ also activates the expression of glucose transporter (GLUT) 1 and GLUT3 and a lactate transporter MCT4. HIF-1 $\alpha$ activates pyruvate dehydrogenase (PDH) kinase 1 , which phosphorylates and inactivates $\mathrm{PDH}$, the enzyme that converts pyruvate to acetyl coenzyme A (acetyl-CoA), which is essential for the TCA cycle.

Warburg and Minami [3] observed almost a century ago that Warburg-type cancer is characterized by the excessive production and accumulation of lactate. Tumors are a heterogeneous collection of normal cells, cancer cells, immune cells, and blood vessels. Even among cancer cells, some are glycolytic and others are oxidative in the context of vascularization and preferred metabolism. Extracellular lactate excreted from glycolytic stromal cells in the tumor tissue may be taken up by adjacent oxidative cancer cells, via MCTs, and be converted to pyruvate to enter the TCA cycle, which produces electron donors, such as $\mathrm{NADH}$ and reduced flavin adenine dinucleotide to support the electron transport chain (ETC) for ATP production. This type of two-compartment communication of tumor metabolism was termed the "reverse Warburg effect" as aerobic glycolysis takes place in tumor stromal fibroblasts rather than cancer cells [59]. The mitochondrial reactive oxygen species (ROS) produced by the ETC activity of reverse Warburg cells are released into the cytosol and inhibit prolyl hydroxylase (PHD) through the Fenton reaction and prevent the degradation of HIF-1 $\alpha$ [60]. The stabilization of HIF- $1 \alpha$ activates the transcription of glycolytic genes, thus converting the oxidative cells to glycolytic ones. Lactate can, in turn, promote HIF- $1 \alpha$ stabilization by inhibiting PHD activity. Lactate-derived pyruvate competitively inhibits alpha-ketoglutarate, a PHD co-factor, from associating with PHD [61]. Recent findings indicate that lactate preconditioning also primes normal fibroblasts to switch from OXPHOS to glycolysis through mechanism(s) including ROS-mediated HIF-1 $\alpha$ stabilization [62]. PKM2 is a rate-limiting enzyme that catalyzes the conversion of phosphoenolpyruvate to pyruvate. PKM2 enzyme activity is allosterically regulated by the oligomerization type. The PKM2 tetramer is enzymatically more active than the dimer form of PKM2. However, the PKM2 dimer acts as a transcriptional coactivator, which may translocate to the nucleus and promote the expression of HIF-1 $\alpha$-mediated pro-glycolytic genes such as lactate-producing LDHA [63]. Thus, lactate, the final product of normoxic glycolysis, further activates glycolysis through the activation of HIF-1 $\alpha$. In addition to hypoxia, the increase in several metabolic intermediates, such as succinate, fumarate, and lactate, facilitates the stabilization of HIF- $1 \alpha$. The oxygen-independent mechanism of HIF-1 $\alpha$ activation is now termed pseudohypoxia [64].

\section{Lactate modulates immune cell functions}

Hypoxia and glycolysis have long been considered to activate the immune cells. For example, a metabolic shift from OXPHOS to aerobic glycolysis is regarded as a hallmark of T cell activation [65]. LDHA induced in activated $\mathrm{T}$ cells to support glycolysis promotes interferon (IFN)- $\gamma$ expression and instead maintains high levels of acetyl-CoA to increase histone acetylation and transcription of ifng [66]. 
Recent studies have highlighted the counterbalancing regulatory function of the glycolysis metabolite lactate. In addition to its metabolic functions as an energy source and an intermediate metabolite for biosynthetic pathways, lactate also plays a modulatory role in inflammation and immunity. Recent reports suggest that lactate produced by aerobic glycolysis has an immunosuppressive effect in the local environment of various disease conditions including sepsis, cancer, chronic inflammation, and autoimmune diseases [6769]. In sepsis, immunosuppression is a serious problem that causes life-threatening secondary infections and is now called immunoparalysis [70]. The immunosuppressive phase during sepsis is characterized by the depletion of effector cells and $\mathrm{T}$ cell exhaustion, and a concomitant increase in regulatory $\mathrm{T}$ cells $\left(\mathrm{T}_{\text {regs }}\right)$ and myeloid-derived suppressor cells [71]. Lactate has also been shown to suppress the proliferation and cytokine production in human cytotoxic T cells [72], thereby decreasing the cytotoxic effect. Effector $\mathrm{T}$ cells are more dependent on glycolysis for proliferation and cytokine production, while $\mathrm{T}_{\text {regs }}$ rely more on OXPHOS [73]. Lactate accumulation in the local tissue environment is a common feature of both inflammatory diseases and cancer. Lactate in the tumor microenvironment (TME) has been shown to help tumor escape from immune surveillance by reshaping $\mathrm{T}$ cells and macrophages to immunosuppressive phenotypes such as tumor-promoting $\mathrm{T}_{\text {regs }}$ and M2-like tumor-associated macrophages (TAMs) [7276].

In contrast, increased lactate concentration observed in the local tissue of chronic inflammatory diseases contributes to the upregulation of the sodium-coupled lactate transporter SLC5A12 by human $\mathrm{CD} 4^{+} \mathrm{T}$ cells. In a mouse arthritis model, lactate was shown to promote $\mathrm{IL}-17$ production by $\mathrm{CD}^{+} \mathrm{T}$ cells through phosphorylation of signal transducer and activator of transcription 3 (STAT3) by PKM2 and fatty acid synthesis (FAS). It also led to $\mathrm{CD}^{+} \mathrm{T}$ cell retention in the inflamed tissue by reducing the glycolysis rate and diverting metabolic fluxes into de novo FAS [68]. Lactate has been shown to inhibit antigen presentation and IL-12 synthesis by dendritic cells (DCs). The differentiation of DCs from monocytes is also affected by high concentrations of lactate, favoring less inflammatory DCs that are biased toward producing IL-10 [76,77].

Macrophages may undergo a switch in the metabolic pathways, which leads to differentiation into either a proinflammatory (M1) or a homeostatic and anti-inflammatory (M2) phenotype in response to various stimuli, including cytokines [78]. In general, IFNs promote M1-like inflammatory macrophage activation by suppressing homeostatic pathways. In the context of tumors, TAMs do not completely follow M1 and M2 subtypes. TAMs usually have an M2-like phenotype and facilitate tumor growth by im- munosuppression. Signaling functions and polarization of M2 macrophages can be directly regulated by lactate in the TME via HIF-1 $\alpha$-dependent metabolic reprogramming [78]. The lactate produced by cancer cells further facilitates HIF-1 $\alpha$ stabilization and induces an M2-like phenotype, such as vascular endothelial growth factor (VEGF). It has been shown that lactate promotes $\mathrm{M} 2$ polarization of macrophages by binding to the lactate receptor HCA1 [48].

\section{Lactate binds to and suppresses mitochondrial antiviral signaling for type I interferon activation}

Viral RNA can be detected by retinoic acid-inducible gene 1 (RIGI)-like receptors (RLRs), such as RIG-I and melanoma differentiation-associated gene 5 (MDA5), which subsequently activate the mitochondrial antiviral signaling (MAVS) protein and downstream axis for type I IFN production [79]. Cells infected by RNA viruses often show an increase in the rate of glycolysis and a decrease in OXPHOS activity, which is a favorable state for rapid viral replication [80]. Furthermore, lactate induced by glycolytic activation inhibits RLR-mediated IFN production [81,82]. Upon recognizing cytosolic double-stranded RNAs, RIG-I undergoes conformational changes, oligomerization, and exposure of the two N-terminal caspase activation and recruitment domains (CARDs) that are involved in a CARD-CARD interaction with MAVS. The transmembrane domain (TM) at the C-terminus of MAVS is required for its localization at the mitochondrial outer membrane. Upon activation, MAVS develops a functional prion-like structure, which serves as a platform for the MAVS signalosome for the activation of the type I IFN pathway. Intriguingly, a recent report showed that lactate inhibits this antiviral axis by directly binding to the TM domain of MAVS, disrupting the mitochondrial localization of MAVS, RIG-I-MAVS interaction and downstream signaling, and IFN- $\beta$ activation [82] (Fig. 1).

\section{Epigenetic lactylation of histones}

Surprisingly, lactate can act as a precursor for the epigenetic lactylation of histone lysine residues, which stimulates gene transcription from chromatin. Histones are proteins that are critical in the packing of DNA into the cell and into chromatin and chromosomes. Histones may experience posttranslational modifications in their protruding $\mathrm{N}$-terminal tails as well as within the $\mathrm{C}$-terminal region. Various histone modifications, including acetylation, methylation, and ubiquitylation, were reported before the discovery of histone lactylation in 2019 [83]. These changes in the amino acids of his- 


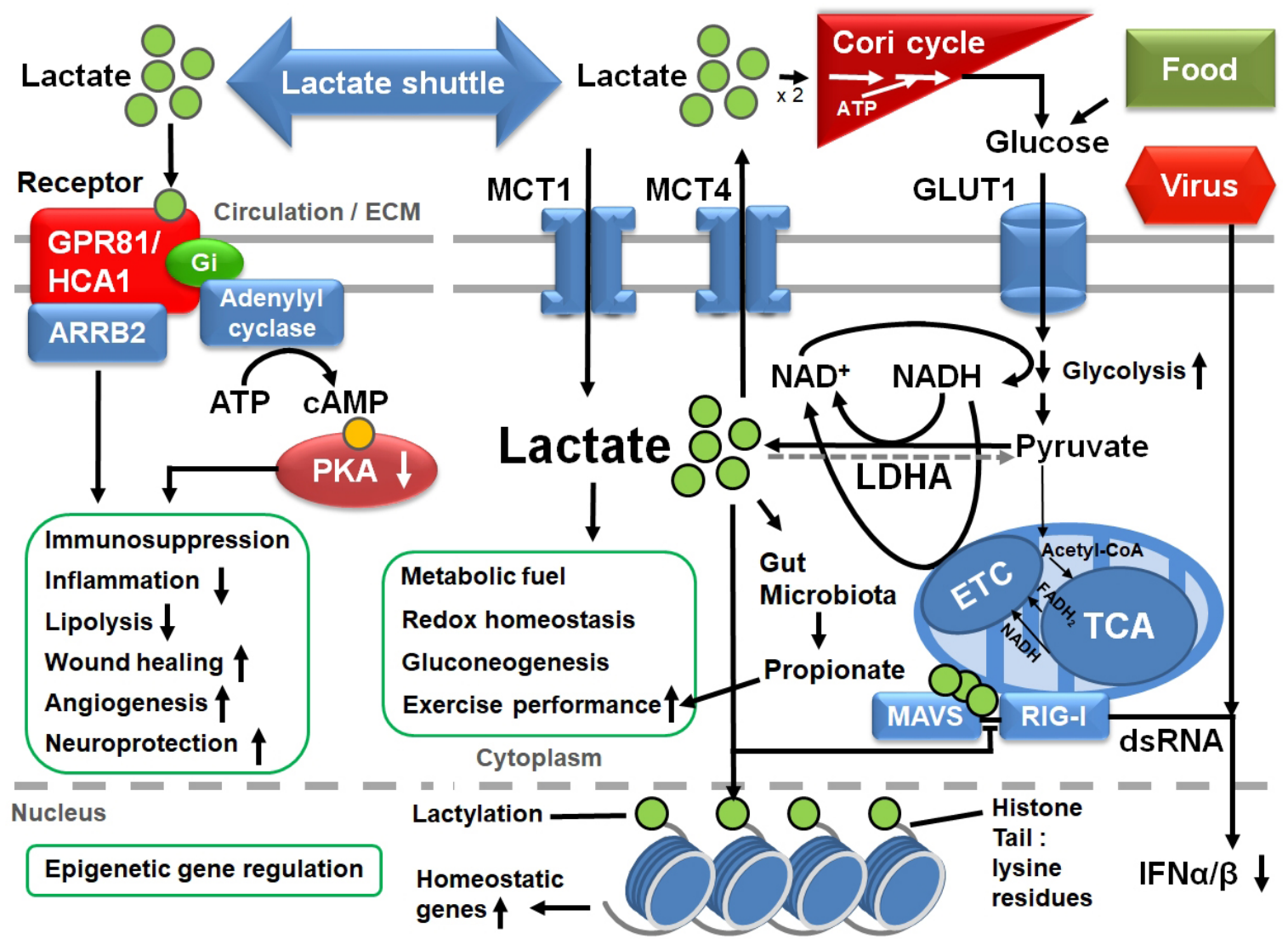

Fig. 1. The metabolism, shuttle, transporters, receptor, and diverse functions (green boxes) of lactate in glycolytic cells. See text for details. GPR81, G-protein coupled receptor 81; HCA1, hydroxycarboxylic acid receptor 1; ECM, extracellular matrix; ARRB2, arrestin beta 2; Gi, inhibitory $\mathrm{G}$ protein; ATP, adenosine triphosphate; CAMP, cyclic adenosine monophosphate; PKA, protein kinase $A_{\text {; }}$ MCT, monocarboxylate transporter; GLUT1, glucose transporter 1 ; NAD, nicotinamide adenine dinucleotide; NADH, reduced form of NAD; LDHA, A form of lactate dehydrogenase (LDH); ETC, electron transport chain; acetyl-CoA, acetyl coenzyme A; FADH2, reduced flavin adenine dinucleotide; TCA, tricarboxylic acid cycle; MAVS, mitochondrial antiviral signaling; RIG-I, retinoic acid-inducible gene 1; dsRNA, double-stranded RNA; IFN, interferon.

tone proteins can affect gene expression as well as overall chromatin condensation. A total of 28 lysine lactylation sites have been identified on the core histones in human and mouse cells. For example, in macrophages stimulated to produce lactate by hypoxia, IFN $\gamma$ plus lipopolysaccharide, or bacterial challenge, histone lactylation accumulated at their promoters is associated with the shift from the initial M1-like phenotype to induction of M2-like homeostatic genes at late time points (16-24 hours) after stimulation. Histone lactylation does not occur at the promoters of proinflammatory genes, such as Tnf or Il6. The induced M2-like genes include Arg1, which is involved in wound healing. Thus, it has been suggested that an endogenous "lactate clock" in M1 macrophages challenged by bacteria turns on the homeostatic genes at the end of the inflammatory phase. This is a new feedback mechanism that restrains macrophage activation [84]. Epigenetic regulation of metabolism and immunity by histone lactylation extends our understanding of the roles of lactate under diverse conditions.

\section{Wound healing}

In healing wounds, cells divide rapidly to activate the glycolytic pathway, resulting in lactate accumulation in the interstitial fluids up to a range of 5-15 $\mathrm{mM}[39,85]$. Lactate has been shown to promote reparative angiogenesis through mechanisms including recruitment of endothelial progenitor cells, stimulation of endothelial cell migration, activation of procollagen factors, and enhancement of collagen deposition in the extracellular matrix. Lactate induces the release of mediators such as VEGF, IL-1, and transforming growth factor beta (TGF- $\beta$ ), all of which consequently stimulate angiogenesis and promote wound healing [86-88]. Exogenous lactate delivery is expected to be helpful in the management of non-healing wounds [88]. 


\section{Microbiome, lactate, propionate, and exercise performance}

The human gut microbiota is linked to various health and disease states. Many studies have indicated that the gut microbiota profile is related to the prevalence of chronic diseases such as diabetes mellitus and metabolic syndrome [89]. Iraporda et al. [90] showed that lactate downregulates proinflammatory responses in intestinal epithelial and myeloid cells. They suggested beneficial effects of lactate-containing foods, such as kefir, on gut microbiota in general and also showed their protective role against infection by pathogenic bacteria such as Salmonella sp. [91,92]. Lactate is an end product of bacterial fermentation in many lactic acid bacteria (i.e., Lactobacillus and Bifidobacterium), which are regarded as key members of the healthy gut microbiota. Lactate produced by $\mathrm{Lac}$ tobacillus and Bifidobacterium in the colon is rapidly converted into short-chain fatty acids, such as propionate, butyrate, and succinate. Lactate produced in the gut may also be released into the systemic circulation via MCTs and removed or metabolized in distant organs [93]. On the other hand, lactate produced by exercising skeletal muscle may be released into circulation and can be transported into the gut lumen where lactate-utilizing microbiota strains are present. This type of novel communication of lactate between the gut and somatic organs was proposed as gut-soma lactate shuttle $[32,33]$.

Many studies on the athlete's gut microbiota have shown distinct microbial compositions, including elevated abundances of Veillonella, Bacteroides, Prevotella, Methanobrevibacter, and Akkermansia $[94,95]$. A bacterial strain belonging to Veillonella atypica was isolated by Scheiman et al. [96] from stool samples of elite marathon runners postmarathon who ran in the 2015 Boston Marathon. Intriguingly, inoculation of this strain into mice significantly increased the treadmill run time by $13 \%$. They found that V.atypica, which utilizes lactate as its sole carbon source, metabolizes lactate into propionate and acetate, as detected by metagenomic analysis. The ${ }^{13} \mathrm{C}_{3}$-labeled lactate tracing experiment in mice showed that serum lactate crosses the epithelial barrier into the lumen of the gut [96]. This study revealed that lactate, a metabolic by-product of aerobic exercise, maybe shuttled into the gut lumen via circulation and provide a carbon source to $V$. atypica, a gut microbiota that can then convert it to a bioactive propionate that improves exercise performance.

\section{Lactate in clinical settings}

Lactate treatment can be helpful in several clinical conditions. For example, lactated Ringer's (LR) solution is one of the most popu- lar crystalloid fluids for patients with trauma, burns, and surgery. LR contains sodium lactate, which is known to be a metabolic fuel that improves cardiac function. In vivo, lactate is rapidly metabolized into bicarbonate by the liver [97]. In a recent study using a neonatal hypoxia-ischemia model, lactate administration reduced the extent of the brain lesion and facilitated behavioral recovery [98]. The usefulness of lactate administration has also been reported in wound healing [88] and muscle regeneration after injury [99].

Increased lactate levels may also be related to maladaptive pathological conditions. For example, lactate accumulation in the TME is associated with cancer progression and poor clinical outcomes, such as resistance to chemotherapy, increased metastasis, and immune evasion $[74,75]$. Lactate is often monitored as a prognostic indicator of severe disease conditions, such as sepsis. It should be noted that increased lactate production is due not only to hypoxia or tissue hypoperfusion but also multiple factors. In addition to hypoxia, serum lactate increases due to increased production during stress conditions (e.g., intense exercise, asthma, and sepsis) and decreased clearance of lactate by the liver and kidney. The lactate levels of patients with asthma or hypertension should be interpreted based on their medication information. Since $\beta 2$-adrenergic stimulators may induce upregulation of lactate production, serum lactate levels can be blunted in patients using $\beta 2$-adrenergic receptor blockers [8].

\section{Conclusion}

Since Warburg's historical finding of aerobic glycolysis in cancer cells, lactate has long been regarded as a culprit of muscle fatigue and acidosis-induced tissue damage. However, it is now accepted that lactate is a useful metabolic fuel for skeletal muscles, heart, and brain. Lactate also functions as a metabolic buffer that links glycolysis and OXPHOS. The introduction of the lactate shuttle concept in the early 1980s vastly changed the lactate paradigm and revealed that lactate is a ubiquitous molecule that is metabolized and used almost everywhere in the body. Lactate shuttles were identified between astrocytes and neurons, contracting and resting skeletal muscles, glycolytic and oxidative cancer cells, and intracellular compartments such as the cytosol and mitochondria. The discovery of the lactate receptor GPR81/HCA1 further extended the landscape of lactate's playing grounds, spanning adipose tissue, skeletal muscles, liver, kidney, heart, brain, immune cells, endothelium, and stromal fibroblasts in the tumor mass. The receptor-mediated nonmetabolic effects of lactate include immunosuppression, active participation in wound healing with increased angiogenesis, decreased adipocyte lipolysis, and neuroprotection 
through anti-inflammatory activities. Lactate overproduced during intense exercise has been shown to cross the epithelial barrier into the gut lumen, increasing the relative abundance of a strain of $V$. atypica. Metabolic conversion of lactate into propionate by $V$. atypi$c a$ has been shown to increase exercise performance. Finally, lactate is involved in the epigenetic regulation of gene expression by lactylating histones, which contributes to immune modulation and maintenance of homeostasis.

Lactate, regarded for a long time as a metabolic waste product since its discovery, is now recognized as a fuel energy source, a precursor of gluconeogenesis, a signaling molecule, a regulator of gene expression, a precursor for exercise-enhancing propionate production by gut microbiota, and probably as more unknowns yet to be explored.

\section{Notes}

\section{Conflicts of interest}

No potential conflict of interest relevant to this article was reported.

\section{ORCID}

Tae-Yoon Lee, https://orcid.org/0000-0003-0437-0765

\section{References}

1. Hsu PP, Sabatini DM. Cancer cell metabolism: Warburg and beyond. Cell 2008;134:703-7.

2. Vander Heiden MG, Cantley LC, Thompson CB. Understanding the Warburg effect: the metabolic requirements of cell proliferation. Science 2009;324:1029-33.

3. Warburg O, Minami S. Versuche an überlebendem carcinom-gewebe. Klin Wochenschr 1923;2:776-7.

4. Warburg O. On the origin of cancer cells. Science 1956;123: 309-14.

5. Ferguson BS, Rogatzki MJ, Goodwin ML, Kane DA, Rightmire Z, Gladden LB. Lactate metabolism: historical context, prior misinterpretations, and current understanding. Eur J Appl Physiol 2018;118:691-728.

6. Hasegawa H, Fukushima T, Lee JA, Tsukamoto K, Moriya K, Ono Y, et al. Determination of serum D-lactic and L-lactic acids in normal subjects and diabetic patients by column-switching HPLC with pre-column fluorescence derivatization. Anal Bioanal Chem 2003;377:886-91.

7. Connor H, Woods HF. Quantitative aspects of L(+)-lactate metabolism in human beings. Ciba Found Symp 1982;87:214 34.
8. Gladden LB. Lactate metabolism: a new paradigm for the third millennium. J Physiol 2004;558:5-30.

9. de la Cruz-López KG, Castro-Muñoz LJ, Reyes-Hernández DO, García-Carrancá A, Manzo-Merino J. Lactate in the regulation of tumor microenvironment and therapeutic approaches. Front Oncol 2019;9:1143.

10. Valvona CJ, Fillmore HL, Nunn PB, Pilkington GJ. The regulation and function of lactate dehydrogenase a: therapeutic potential in brain tumor. Brain Pathol 2016;26:3-17.

11. Fletcher WM. Lactic acid in amphibian muscle. J Physiol 1907; 35:247-309.

12. Hill AV, Long CN, Lupton H. Muscular exercise, lactic acid, and the supply and utilisation of oxygen. Proc R Soc Lond B Contain Pap Biol Character 1924;97:84-138.

13. Meyerhof $O$. The chemistry of muscular contraction. Lancet $1930 ; 216: 1415-22$.

14. Wasserman $\mathrm{K}$. The anaerobic threshold measurement to evaluate exercise performance. Am Rev Respir Dis 1984;129(2 Pt 2):S35-40.

15. Garcia-Alvarez M, Marik P, Bellomo R. Sepsis-associated hyperlactatemia. Crit Care 2014;18:503.

16. Kraut JA, Madias NE. Lactic acidosis. N Engl J Med 2014;371: 2309-19.

17. Robergs RA, Ghiasvand F, Parker D. Biochemistry of exercise-induced metabolic acidosis. Am J Physiol Regul Integr Comp Physiol 2004;287:R502-16.

18. Feher JJ. Quantitative human physiology: an introduction. 2.9 ATP production I: glycolysis. 2nd ed. International: Elsevier Science Publishing; 2016. p. 171-9.

19. Argiles A, Mourad G, Mion C, Atkins RC, Haiech J. Kidney, proteins and drugs. In: Bianchi C, Bocci V, Carone FA, Rabkin R, editors. 6th International Symposium on Nephrology, Montecatini Terme. Basel: Karger; 1990.p. 1-8.

20. Levraut J, Ciebiera JP, Jambou P, Ichai C, Labib Y, Grimaud D. Effect of continuous venovenous hemofiltration with dialysis on lactate clearance in critically ill patients. Crit Care Med 1997; $25: 58-62$.

21. Cori CF, Cori GT. The carbohydrate metabolism of tumors. J Biol Chem 1925;65:397-405.

22. Consoli A, Nurjhan N, Reilly JJ Jr, Bier DM, Gerich JE. Contribution of liver and skeletal muscle to alanine and lactate metabolism in humans. Am J Physiol 1990;259:E677-84.

23. Miller BF, Fattor JA, Jacobs KA, Horning MA, Navazio F, Lindinger MI, et al. Lactate and glucose interactions during rest and exercise in men: effect of exogenous lactate infusion. J Physiol 2002;544:963-75.

24. Brooks GA. Lactate: glycolytic end product and oxidative sub- 
strate during sustained exercise in mammals - the "lactate shuttle”. In: Gilles R, editor. Comparative physiology and biochemistry: current topics and trends. Vol. A. Respiration-metabolism-circulation. Berlin: Springer-Verlag; 1985. p. 208-18.

25. Brooks GA. Anaerobic threshold: review of the concept and directions for future research. Med Sci Sports Exerc 1985;17:2234.

26. Brooks GA. Lactate production under fully aerobic conditions: the lactate shuttle during rest and exercise. Fed Proc 1986;45: 2924-9.

27. Brooks GA. Lactate shuttles in nature. Biochem Soc Trans 2002;30:258-64.

28. Boussouar F, Benahmed M. Lactate and energy metabolism in male germ cells. Trends Endocrinol Metab 2004;15:345-50.

29. Ahmed K, Tunaru S, Tang C, Müller M, Gille A, Sassmann A, et al. An autocrine lactate loop mediates insulin-dependent inhibition of lipolysis through GPR81. Cell Metab 2010;11:311-9.

30. Liu L, MacKenzie KR, Putluri N, Maletić-Savatić M, Bellen HJ. The glia-neuron lactate shuttle and elevated ROS promote lipid synthesis in neurons and lipid droplet accumulation in glia via APOE/D. Cell Metab 2017;26:719-37.

31. Johnson ML, Hussien R, Horning MA, Brooks GA. Transpulmonary pyruvate kinetics. Am J Physiol Regul Integr Comp Physiol 2011;301:R769-74.

32. Brooks GA. The science and translation of lactate shuttle theory. Cell Metab 2018;27:757-85.

33. Brooks GA. Lactate as a fulcrum of metabolism. Redox Biol 2020;35:101454.

34. Mazzeo RS, Brooks GA, Schoeller DA, Budinger TF. Disposal of blood [1-13C] lactate in humans during rest and exercise. J Appl Physiol (1985) 1986;60:232-41.

35. Hashimoto T, Hussien R, Brooks GA. Colocalization of MCT1, CD147, and LDH in mitochondrial inner membrane of L6 muscle cells: evidence of a mitochondrial lactate oxidation complex. Am J Physiol Endocrinol Metab 2006;290:E123744.

36. Stanley WC. Myocardial lactate metabolism during exercise. Med Sci Sports Exerc 1991;23:920-4.

37. van Hall G. Lactate kinetics in human tissues at rest and during exercise. Acta Physiol (Oxf) 2010;199:499-508.

38. Pellerin L, Pellegri G, Bittar PG, Charnay Y, Bouras C, Martin $\mathrm{JL}$, et al. Evidence supporting the existence of an activity-dependent astrocyte-neuron lactate shuttle. Dev Neurosci 1998;20: 291-9.

39. Sun S, Li H, Chen J, Qian Q. Lactic acid: no longer an inert and end-product of glycolysis. Physiology (Bethesda) 2017;32: 453-63.
40. Halestrap AP, Price NT. The proton-linked monocarboxylate transporter (MCT) family: structure, function and regulation. Biochem J 1999;343 Pt 2:281-99.

41. Srinivas SR, Gopal E, Zhuang L, Itagaki S, Martin PM, Fei YJ, et al. Cloning and functional identification of slc5a 12 as a sodium-coupled low-affinity transporter for monocarboxylates (SMCT2). Biochem J 2005;392:655-64.

42. Haas R, Smith J, Rocher-Ros V, Nadkarni S, Montero-Melendez T, D'Acquisto F, et al. Lactate regulates metabolic and pro-inflammatory circuits in control of $\mathrm{T}$ cell migration and effector functions. PLoS Biol 2015;13:e1002202.

43. Liu C, Kuei C, Zhu J, Yu J, Zhang L, Shih A, et al. 3,5-Dihydroxybenzoic acid, a specific agonist for hydroxycarboxylic acid 1, inhibits lipolysis in adipocytes. J Pharmacol Exp Ther 2012; 341:794-801.

44. de Castro Abrantes H, Briquet M, Schmuziger C, Restivo L, Puyal J, Rosenberg N, et al. The lactate receptor HCAR1 modulates neuronal network activity through the activation of $\mathrm{G} \alpha$ and G $\beta$ r subunits. J Neurosci 2019;39:4422-33.

45. Ohno Y, Oyama A, Kaneko H, Egawa T, Yokoyama S, Sugiura T, et al. Lactate increases myotube diameter via activation of MEK/ERK pathway in C2C12 cells. Acta Physiol (Oxf) 2018;223:e13042.

46. Roland CL, Arumugam T, Deng D, Liu SH, Philip B, Gomez S, et al. Cell surface lactate receptor GPR81 is crucial for cancer cell survival. Cancer Res 2014;74:5301-10.

47. Harun-Or-Rashid M, Inman DM. Reduced AMPK activation and increased HCAR activation drive anti-inflammatory response and neuroprotection in glaucoma. J Neuroinflammation 2018;15:313.

48. Hoque R, Farooq A, Ghani A, Gorelick F, Mehal WZ. Lactate reduces liver and pancreatic injury in Toll-like receptor- and inflammasome-mediated inflammation via GPR81-mediated suppression of innate immunity. Gastroenterology 2014;146: 1763-74.

49. Brown TP, Ganapathy V. Lactate/GPR81 signaling and proton motive force in cancer: role in angiogenesis, immune escape, nutrition, and Warburg phenomenon. Pharmacol Ther 2020; 206:107451.

50. Langin D. Adipose tissue lipolysis revisited (again!): lactate involvement in insulin antilipolytic action. Cell Metab 2010;11: 242-3.

51. Liu C, Wu J, Zhu J, Kuei C, Yu J, Shelton J, et al. Lactate inhibits lipolysis in fat cells through activation of an orphan G-protein-coupled receptor, GPR81. J Biol Chem 2009;284:281122.

52. Khatib-Massalha E, Bhattacharya S, Massalha H, Biram A, Go- 
lan K, Kollet O, et al. Lactate released by inflammatory bone marrow neutrophils induces their mobilization via endothelial GPR81 signaling. Nat Commun 2020;11:3547.

53. Hu J, Cai M, Liu Y, Liu B, Xue X, Ji R, et al. The roles of GRP81 as a metabolic sensor and inflammatory mediator. J Cell Physiol 2020;235:8938-50.

54. Otto AM. Warburg effect(s)-a biographical sketch of Otto Warburg and his impacts on tumor metabolism. Cancer Metab 2016;4:5

55. Racker E. Bioenergetics and the problem of tumor growth. Am Sci 1972;60:56-63.

56. Bonnet S, Archer SL, Allalunis-Turner J, Haromy A, Beaulieu C, Thompson $\mathrm{R}$, et al. A mitochondria- $\mathrm{K}+$ channel axis is suppressed in cancer and its normalization promotes apoptosis and inhibits cancer growth. Cancer Cell 2007;11:37-51.

57. DeBerardinis RJ, Chandel NS. We need to talk about the Warburg effect. Nat Metab 2020;2:127-9.

58. Semenza GL, Wang GL. A nuclear factor induced by hypoxia via de novo protein synthesis binds to the human erythropoietin gene enhancer at a site required for transcriptional activation. Mol Cell Biol 1992;12:5447-54.

59. Pavlides S, Whitaker-Menezes D, Castello-Cros R, Flomenberg N, Witkiewicz AK, Frank PG, et al. The reverse Warburg effect: aerobic glycolysis in cancer associated fibroblasts and the tumor stroma. Cell Cycle 2009;8:3984-4001.

60. De Saedeleer CJ, Copetti T, Porporato PE, Verrax J, Feron O, Sonveaux P. Lactate activates HIF-1 in oxidative but not in Warburg-phenotype human tumor cells. PLoS One 2012;7: e46571.

61. Ali MA, Konishi T. Enhancement of hydroxyl radical generation in the Fenton reaction by alpha-hydroxy acid. Biochem Mol Biol Int 1998;46:137-45.

62. Kozlov AM, Lone A, Betts DH, Cumming RC. Lactate preconditioning promotes a HIF-1 $\alpha$-mediated metabolic shift from OXPHOS to glycolysis in normal human diploid fibroblasts. Sci Rep 2020;10:8388.

63. Luo W, Semenza GL. Pyruvate kinase M2 regulates glucose metabolism by functioning as a coactivator for hypoxia-inducible factor 1 in cancer cells. Oncotarget 2011;2:551-6.

64. Hayashi Y, Yokota A, Harada H, Huang G. Hypoxia/pseudohypoxia-mediated activation of hypoxia-inducible factor- $1 \alpha$ in cancer. Cancer Sci 2019;110:1510-7.

65. Chang CH, Curtis JD, Maggi LB Jr, Faubert B, Villarino AV, O'Sullivan D, et al. Posttranscriptional control of T cell effector function by aerobic glycolysis. Cell 2013;153:1239-51.

66. Peng M, Yin N, Chhangawala S, Xu K, Leslie CS, Li MO. Aerobic glycolysis promotes $\mathrm{T}$ helper 1 cell differentiation through an epigenetic mechanism. Science 2016;354:481-4.

67. Nolt B, Tu F, Wang X, Ha T, Winter R, Williams DL, et al. Lactate and Immunosuppression in Sepsis. Shock 2018;49:120-5.

68. Pucino V, Bombardieri M, Pitzalis C, Mauro C. Lactate at the crossroads of metabolism, inflammation, and autoimmunity. Eur J Immunol 2017;47:14-21.

69. Hirschhaeuser F, Sattler UG, Mueller-Klieser W. Lactate: a metabolic key player in cancer. Cancer Res 2011;71:6921-5.

70. Hotchkiss RS, Monneret G, Payen D. Sepsis-induced immunosuppression: from cellular dysfunctions to immunotherapy. Nat Rev Immunol 2013;13:862-74.

71. Hotchkiss RS, Monneret G, Payen D. Immunosuppression in sepsis: a novel understanding of the disorder and a new therapeutic approach. Lancet Infect Dis 2013;13:260-8.

72. Fischer K, Hoffmann P, Voelkl S, Meidenbauer N, Ammer J, Edinger M, et al. Inhibitory effect of tumor cell-derived lactic acid on human T cells. Blood 2007;109:3812-9.

73. Michalek RD, Gerriets VA, Jacobs SR, Macintyre AN, MacIver NJ, Mason EF, et al. Cutting edge: distinct glycolytic and lipid oxidative metabolic programs are essential for effector and regulatory CD4+ T cell subsets. J Immunol 2011;186:3299-303.

74. Wenes M, Shang M, Di Matteo M, Goveia J, Martín-Pérez R, Serneels J, et al. Macrophage metabolism controls tumor blood vessel morphogenesis and metastasis. Cell Metab 2016;24: $701-15$.

75. Certo M, Tsai CH, Pucino V, Ho PC, Mauro C. Lactate modulation of immune responses in inflammatory versus tumour microenvironments. Nat Rev Immunol 2021;21:151-61.

76. Nasi A, Rethi B. Disarmed by density: a glycolytic break for immunostimulatory dendritic cells? Oncoimmunology 2013; 2:e26744.

77. Gottfried E, Kunz-Schughart LA, Ebner S, Mueller-Klieser W, Hoves S, Andreesen R, et al. Tumor-derived lactic acid modulates dendritic cell activation and antigen expression. Blood 2006;107:2013-21.

78. Kelly B, O'Neill LA. Metabolic reprogramming in macrophages and dendritic cells in innate immunity. Cell Res 2015;25:77184.

79. Schlee M, Hartmann G. Discriminating self from non-self in nucleic acid sensing. Nat Rev Immunol 2016;16:566-80.

80. Ivashkiv LB. The hypoxia-lactate axis tempers inflammation. Nat Rev Immunol 2020;20:85-6.

81. Yasukawa K, Kinoshita D, Yaku K, Nakagawa T, Koshiba T. The microRNAs miR-302b and miR-372 regulate mitochondrial metabolism via the SLC25A12 transporter, which controls MAVS-mediated antiviral innate immunity. J Biol Chem 2020;295:444-57. 
82. Zhang W, Wang G, Xu ZG, Tu H, Hu F, Dai J, et al. Lactate is a natural suppressor of RLR signaling by targeting MAVS. Cell 2019; 178:176-89.

83. Prakash K, Fournier D. Evidence for the implication of the histone code in building the genome structure. Biosystems 2018; 164:49-59.

84. Zhang D, Tang Z, Huang H, Zhou G, Cui C, Weng Y, et al. Metabolic regulation of gene expression by histone lactylation. Nature 2019;574:575-80.

85. Hunt TK, Conolly WB, Aronson SB, Goldstein P. Anaerobic metabolism and wound healing: an hypothesis for the initiation and cessation of collagen synthesis in wounds. Am J Surg 1978;135:328-32.

86. Constant JS, Feng JJ, Zabel DD, Yuan H, Suh DY, Scheuenstuhl $\mathrm{H}$, et al. Lactate elicits vascular endothelial growth factor from macrophages: a possible alternative to hypoxia. Wound Repair Regen 2000;8:353-60.

87. Hunt TK, Aslam RS, Beckert S, Wagner S, Ghani QP, Hussain MZ, et al. Aerobically derived lactate stimulates revascularization and tissue repair via redox mechanisms. Antioxid Redox Signal 2007;9:1115-24.

88. Porporato PE, Payen VL, De Saedeleer CJ, Préat V, Thissen JP, Feron $\mathrm{O}$, et al. Lactate stimulates angiogenesis and accelerates the healing of superficial and ischemic wounds in mice. Angiogenesis 2012;15:581-92.

89. Turnbaugh PJ, Ley RE, Hamady M, Fraser-Liggett CM, Knight R, Gordon JI. The human microbiome project. Nature 2007; 449:804-10.

90. Iraporda C, Errea A, Romanin DE, Cayet D, Pereyra E, Pignataro $\mathrm{O}$, et al. Lactate and short chain fatty acids produced by microbial fermentation downregulate proinflammatory responses in intestinal epithelial cells and myeloid cells. Immunobiology 2015;220:1161-9.

91. Iraporda C, Romanin DE, Rumbo M, Garrote GL, Abraham
AG. The role of lactate on the immunomodulatory properties of the nonbacterial fraction of kefir. Food Res Int 2014;62:24753.

92. Iraporda C, Abatemarco Júnior M, Neumann E, Nunes ÁC, Nicoli JR, Abraham AG, et al. Biological activity of the non-microbial fraction of kefir: antagonism against intestinal pathogens. J Dairy Res 2017;84:339-45.

93. Teramae H, Yoshikawa T, Inoue R, Ushida K, Takebe K, Nio-Kobayashi J, et al. The cellular expression of SMCT2 and its comparison with other transporters for monocarboxylates in the mouse digestive tract. Biomed Res 2010;31:239-49.

94. Clarke SF, Murphy EF, O'Sullivan O, Lucey AJ, Humphreys M, Hogan A, et al. Exercise and associated dietary extremes impact on gut microbial diversity. Gut 2014;63:1913-20.

95. Petersen LM, Bautista EJ, Nguyen H, Hanson BM, Chen L, Lek $\mathrm{SH}$, et al. Community characteristics of the gut microbiomes of competitive cyclists. Microbiome 2017;5:98.

96. Scheiman J, Luber JM, Chavkin TA, MacDonald T, Tung A, Pham LD, et al. Meta-omics analysis of elite athletes identifies a performance-enhancing microbe that functions via lactate metabolism. Nat Med 2019;25:1104-9.

97. Nalos M, Leverve X, Huang S, Weisbrodt L, Parkin R, Seppelt I, et al. Half-molar sodium lactate infusion improves cardiac performance in acute heart failure: a pilot randomised controlled clinical trial. Crit Care 2014;18:R48.

98. Tassinari ID, Andrade MKG, da Rosa LA, Hoff ML, Nunes RR, Vogt EL, et al. Lactate administration reduces brain injury and ameliorates behavioral outcomes following neonatal hypoxia-ischemia. Neuroscience 2020;448:191-205.

99. Zhang J, Muri J, Fitzgerald G, Gorski T, Gianni-Barrera R, Masschelein E, et al. Endothelial lactate controls muscle regeneration from ischemia by inducing $\mathrm{m} 2$-like macrophage polarization. Cell Metab 2020;31:1136-53. 